\title{
MODELAGEM GEOESTATÍSTICA DA ESTRUTURA ESPACIAL ARBÓREA E DOS ATRIBUTOS DO SOLO EM FLORESTA OMBRÓFILA MISTA
}

\author{
Marcelo Roveda ${ }^{1 *}$, Allan Libanio Pelissari², Aline Marques Genú ${ }^{3}$, Afonso Figueiredo Filho ${ }^{4}$, Mailson Roik ${ }^{5}$ \\ 1* Universidade Federal do Paraná, Programa de Pós-Graduação em Engenharia Florestal, Curitiba, Paraná, Brasil - \\ marcelo_roveda@hotmail.com \\ ${ }^{2}$ Universidade Federal do Paraná, Departamento de Ciências Florestais, Curitiba, Paraná, Brasil - allanpelissari@ gmail.com \\ ${ }^{3}$ Universidade Estadual do Centro Oeste, Departamento de Agronomia, Guarapuava, Paraná, Brasil - amgenu@ gmail.com \\ ${ }^{4}$ Universidade Federal do Paraná, Programa de Pós-Graduação em Engenharia Florestal, Curitiba, Paraná, Brasil - afigfilho@ gmail.com \\ ${ }^{5}$ Universidade Federal do Paraná, Programa de Pós-Graduação em Engenharia Florestal, Curitiba, Paraná, Brasil - \\ mailsonroik@hotmail.com
}

Recebido para publicação: 19/01/2017 - Aceito para publicação: 08/02/2018

\begin{abstract}
Resumo
Considerando a hipótese de que a geoestatística possibilita aprimorar o entendimento da variabilidade espacial das formações florestais naturais e de suas características edáficas, o objetivo deste trabalho foi avaliar as relações espaciais entre os atributos químicos e granulométricos do solo sobre o número de árvores e a área basal de um remanescente de Floresta Ombrófila Mista. O levantamento dos atributos do solo e da vegetação foram realizados em 200 unidades de amostra georreferenciadas de 25 x $25 \mathrm{~m}$. Em seguida, a geoestatística foi utilizada para modelar a continuidade espacial, e a krigagem ordinária pontual foi aplicada para gerar os mapas temáticos. Por meio dos resultados obtidos, confirmou-se que a continuidade espacial dos atributos do solo, número de árvores e área basal são passíveis de serem modeladas, ao passo que não foi observado similaridade espacial nos mapas de krigagem. Entretanto, ficou evidente que, entre as variáveis edáficas consideradas, o pH possui a maior relação espacial com as variáveis da floresta.

Palavras-chave: Floresta com araúcaria, semivariograma, krigagem.
\end{abstract}

\begin{abstract}
Geostatistical modeling of tree spatial structure and soil attributes in Mixed Ombrophilous Forest. Considering the hypothesis that geostatistics provides improvement for understanding the spatial variability of natural forest formations and their soil characteristics, the aim of this study was to evaluate the spatial relationship between the soil chemical and granulometric attributes regarding the number of trees and the basal area of a Mixed Ombrophilous Forest remnant. The survey of soil attributes and vegetation was carried out in 200 units of $25 \mathrm{x}$ $25 \mathrm{~m}$ georeferenced sampling units. Then, geostatistics was used to model the spatial continuity, and punctual ordinary kriging was employed to produce thematic maps. The results obtained confirmed that the spatial continuity of soil attributes, number of trees and basal area can be modelled, whreas non-spatial similarity was observed in the kriging maps. However, it was evident that among the soil variables considered, $\mathrm{pH}$ is the one with the greatest spatial relationship with the forest variables.

Keywords: Araucaria forest, semivariogram, kriging.
\end{abstract}

\section{INTRODUÇÃO}

Nas últimas décadas, observou-se um crescente número de levantamentos florísticos para a caracterização da vegetação de diferentes formações florestais com o objetivo de conservar a biodiversidade e o manejo florestal (CARVALHO et al., 2007). Todavia, notou-se que as características quantitativas e qualitativas da estrutura das florestas são condicionadas aos atributos do solo (LOPES et al., 2016).

O solo é um importante constituinte dos ecossistemas florestais que influencia no desenvolvimento das florestas naturais (RODRIGUES et al., 2016) à medida que fornece nutrientes às árvores, retém a água e abriga a fauna. Além disso, ao longo do tempo, a floresta atua no solo por meio de seu sistema radicular e pela deposição de material orgânico (RAIJ, 2011). Entre os atributos do solo, a granulometria é a característica que mais influencia na capacidade produtiva dos sítios florestais (KITAMURA et al., 2007). Ela está diretamente relacionada com a estrutura do solo, a consistência, a capacidade de retenção de água, a drenagem e as reações de troca entre o sistema radicular e o complexo coloidal, ao passo que as condições químicas do solo garantem a disponibilidade de nutrientes às plantas em desenvolvimento (SILVA et al., 2012). 
As características edáficas são fenômenos naturais que se distribuem com algum grau de organização ou continuidade, sendo expressa pela dependência espacial à medida que as análises estatísticas detectam as variações entre as unidades amostrais que ocorrem de forma casual, independente e normalmente distribuída (VIEIRA et al., 1997). Sendo assim, as técnicas baseadas na estatística espacial, como a geoestatística, permitem detectar a existência da dependência espacial, constituindo-se em uma importante ferramenta na análise e na descrição detalhada do comportamento das variáveis do ambiente (YAMAMOTO; LANDIM, 2013).

Por meio dos mapas elaborados por geoestatística, é possível inferir sobre a relação das variáveis dendrométricas da vegetação com os atributos do solo e do relevo, visando identificar as principais limitações à produção e evitar a exaustão e a degradação do solo (RUFINO et al., 2006). Dessa forma, a caracterização espacial é fundamental para o entendimento dos ecossistemas florestais, em que muitos estudos objetivam investigar as relações entre a distribuição e o crescimento de florestas nativas e suas relações com as variáveis do ambiente (SANTOS et al., 2013a).

Estudos da relação entre o levantamento arbóreo e edáfico utilizando estatísticas de medidas de tendência central, medidas de dispersão e multivariadas foram realizados por diversos autores, destacando-se os trabalhos de: Gerhardt et al. (2001) em povoamento de Araucaria angustifolia localizado na Floresta Nacional de Canela, estado do Rio Grande do Sul; Carvalho et al. (2007) em fragmento de Floresta Estacional Semidecidual em Piedade do Rio Grande, estado de Minas Gerais; Toledo et al. (2009) em ambientes de cerrado no Norte de Minas Gerais; Rovedder et al. (2014) em remanescente da Floresta Estacional Decidual na Região Central do Rio Grande do Sul; e Rodrigues et al. (2016) em Floresta Ombrófila Mista no município de Guarapuava, estado do Paraná. Contudo, em relação ao uso da geoestatística para atributos do solo e variáveis dendrométricas de florestas nativas, há a necessidade de pesquisas indicadoras para mapeamento e investigação das possíveis relações espaciais.

Assim, considerando a hipótese de que a geoestatística possibilita aprimorar o entendimento da variabilidade espacial das formações florestais naturais e de suas características edáficas, o objetivo do presente trabalho foi avaliar a dependência espacial dos atributos químicos e granulométricos do solo e suas relações espaciais com o número de árvores e a área basal de um remanescente de Floresta Ombrófila Mista.

\section{MATERIAL E MÉTODOS}

A pesquisa foi conduzida em um remanescente de Floresta Ombrófila Mista Montana localizado na Floresta Nacional de Irati, a uma altitude média de $820 \mathrm{~m}$, entre as coordenadas $25^{\circ} 01^{\prime} \mathrm{S}$ e $25^{\circ} 40^{\prime} \mathrm{S}$ e $51^{\circ} 11^{\prime} \mathrm{O}$ e $51^{\circ} 15^{\prime} \mathrm{O}$, com clima Cfb (Köppen), temperatura média de $17,5^{\circ} \mathrm{C}$ e pluviosidade superior a $1.500 \mathrm{~mm}^{\mathrm{ano}}{ }^{-1}$. Com a descrição morfológica (SANTOS et al., 2013b) e a classificação do solo (EMBRAPA, 2013) para a área de estudo, foram identificadas cinco classes de solo: Latossolo Vermelho distrófico típico (LVd), Latossolo Amarelo distrófico típico (LAd), Cambissolo Háplico Ta distrófico típico (CXvdt), Cambissolo Háplico Ta distrófico léptico (CXvdl) e Cambissolo Háplico alítico típico (CXal).

A área do remanescente foi segmentada em uma malha sistemática composta por 200 unidades de amostras de $25 \times 25 \mathrm{~m}$. Para cada uma, foram determinados o número de árvores $\left(\mathrm{n} \mathrm{ha}^{-1}\right)$ e a área basal $\left(\mathrm{m}^{2} \mathrm{ha}^{-1}\right)$ de todos os indivíduos arbóreos (DAP $\geq 10 \mathrm{~cm}$ ) e coletada uma amostra simples de solo na profundidade de 0 - 0,20 m no centro de cada unidade de amostra (Figura 1), que foi submetida a análise no Laboratório de Solos da Universidade Estadual do Centro-Oeste para determinação do $\mathrm{pH}$ (em $\left.\mathrm{CaCl}_{2}\right)$, fósforo $(\mathrm{P}$ - solução de $\mathrm{Mehlich}$ ), potássio $(\mathrm{K})$, cálcio $(\mathrm{Ca})$, magnésio $(\mathrm{Mg})$, alumínio $(\mathrm{Al})$, hidrogênio e alumínio $(\mathrm{H}+\mathrm{Al})$ e granulometria (areia, silte e argila) pelo método da pipeta, conforme metodologias da Embrapa (2013).

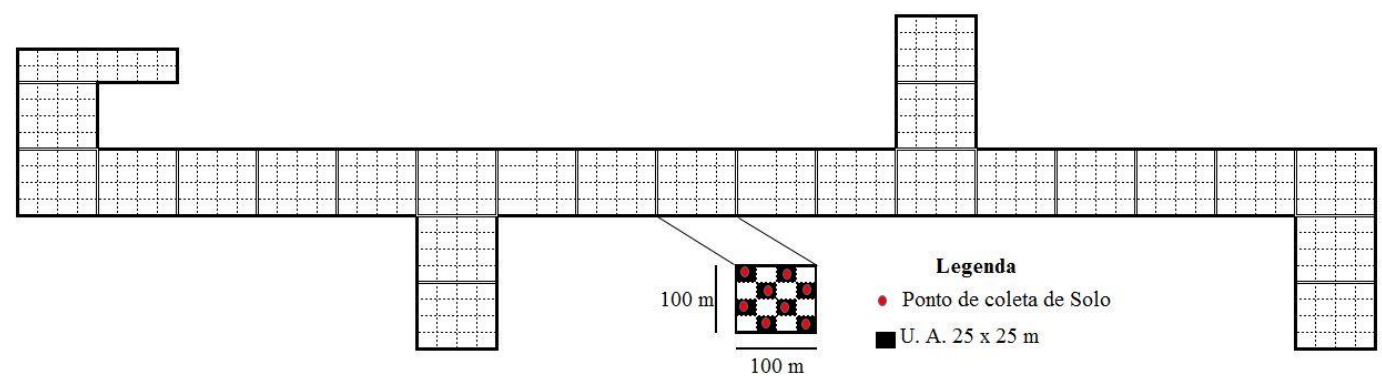

Figura 1. Alocação dos pontos de coleta de solo e das unidades amostrais para o remanescente de Floresta Ombrófila Mista. Figure 1. Allocation of soil points and sampling units in a Mixed Ombrophilous Forest remnant.

Os dados foram submetidos à análise estatística descritiva, ao teste de outliers de Grubbs e à hipótese de normalidade pelo teste Kolmogorov-Smirnov, ambos para a probabilidade de $95 \%$. O comportamento da assimetria foi utilizado para avaliar a necessidade de transformação dos dados, conforme proposto por Webster e Oliver (2007). 
Após a avaliação exploratória, a análise geoestatística foi aplicada para a identificação da possível dependência espacial do número de árvores, da área basal e dos atributos do solo, por meio da determinação da semivariância (1) considerando o posicionamento geográfico das unidades de amostras no campo e o posterior cômputo das distâncias (h) e das diferenças numéricas da variável (Z) na malha de pontos (ISAAKS; SRIVASTAVA, 1989).

$$
\gamma(\mathrm{h})=\frac{1}{2 \mathrm{~N}(\mathrm{~h})} \sum_{\mathrm{i}=1}^{\mathrm{N}(\mathrm{h})}\left[\mathrm{Z}\left(\mathrm{x}_{\mathrm{i}}\right)-\mathrm{Z}\left(\mathrm{x}_{\mathrm{i}}+\mathrm{h}\right)\right]^{2}
$$

em que: $\gamma(\mathrm{h})=$ semivariância da variável $\mathrm{Z}\left(\mathrm{x}_{\mathrm{i}}\right) ; \mathrm{h}=$ distância $(\mathrm{m})$; e $\mathrm{N}(\mathrm{h})=$ número de pares de pontos medidos $\mathrm{Z}\left(\mathrm{x}_{\mathrm{i}}\right)$ e $\mathrm{Z}\left(\mathrm{x}_{\mathrm{i}}+\mathrm{h}\right)$, separados por uma distância $\mathrm{h}$.

Os semivariogramas foram escalonados visando padronizar a comparação entre as diferentes variáveis, conforme recomendado por Vieira et al. (1997). Além disso, as semivariâncias foram determinadas entre os pontos das unidades de amostra equidistantes, com a regularização da malha amostral por meio de uma tolerância angular de $22,5^{\circ}$. Esse processo foi repetido em quatro direções no plano espacial: $0^{\circ}(\mathrm{S}-\mathrm{N}) ; 45^{\circ}(\mathrm{SO}-\mathrm{NE}) ; 90^{\circ}(\mathrm{O}-\mathrm{L})$; e $135^{\circ}(\mathrm{NO}-\mathrm{SE})$, dos quais foi obtida a matriz das semivariâncias médias entre as distâncias equivalentes (YAMAMOTO; LANDIM, 2013).

Os modelos esférico, exponencial e gaussiano foram utilizados para descrever a estrutura da dependência espacial por meio do programa $\mathrm{GS}^{+}$. Para seu ajuste, a estrutura do semivariograma teórico foi definida, segundo Yamamoto e Landim (2013), como: $C_{0}=$ efeito pepita (valor da semivariância para a distância zero, a qual representa o componente da variação ao acaso); $\mathrm{C}=$ contribuição (variância estrutural); $\mathrm{C}_{0}+\mathrm{C}=$ patamar (valor da semivariância em que a curva se estabiliza em um valor constante); e $\mathrm{A}=$ alcance (distância da origem até o ponto em que o patamar atinge valores estáveis).

O ajuste dos semivariogramas teóricos foi efetuado pelo Método dos Quadrados Mínimos Ponderados, com a avaliação e a seleção dos modelos efetuada com base na menor soma de quadrados dos desvios ponderados (SQDP), no maior coeficiente de determinação $\left(\mathrm{R}^{2}\right)$ e na validação cruzada (ISAAKS; SRIVASTAVA, 1989), a qual considera os coeficientes lineares, angulares e de determinação da validação cruzada $\left(\mathrm{R}^{2}{ }_{\mathrm{vc}}\right)$ e o erro padrão estimativa $\left(\mathrm{S}_{\mathrm{yx}} \%\right)$.

Para analisar o grau de dependência espacial (GD), utilizou-se a classificação de Cambardella et al. (1994), em que os semivariogramas com efeito pepita menor ou igual a $25 \%$ do patamar foram considerados com GD forte; moderados, quando GD entre 25 e $75 \%$; e fracos, quando GD maior que $75 \%$. Posteriormente, a interpolação foi efetuada por meio da krigagem ordinária pontual (Equação 2), que consiste em um estimador linear não enviesado e com mínima variância para a interpolação em posições não amostradas (ISAAKS; SRIVASTAVA, 1989), em que os pesos $\left(\lambda_{\mathrm{i}}\right.$ foram determinados pela técnica de multiplicadores de Lagrange (WEBSTER; OLIVER, 2007). Em seguida, os mapas temáticos foram feitos com o auxílio do programa computacional $\mathrm{GS}^{+}$e as classes determinadas conforme os quartis de cada variável.

$$
Z_{K O}\left(x_{0}\right)=\sum_{i=1}^{n} \lambda_{i}\left[Z\left(x_{i}\right)\right]
$$

em que: $Z_{K O}=$ estimador de krigagem ordinária; $\lambda_{i}=$ peso; $Z\left(x_{i}\right)=$ dados experimentais; e $n=$ número de dados.

\section{RESULTADOS}

Por meio do teste de Grubbs, foi observada a presença de outliers para as variáveis pH, $\mathrm{P}, \mathrm{K}$, Ca e $\mathrm{Mg}$ (Tabela 1), sendo realizada a remoção a fim de obter melhoria na distribuição dos dados. Para as demais variáveis, não foram observados outliers nas bases de dados, acarretando na não rejeição da hipótese de nulidade. Ao analisar as medidas de dispersão dos atributos do solo, os maiores coeficientes de variação (CV) foram observados para os atributos químicos, exceto para o $\mathrm{pH}$. Para a granulometria, os maiores desvios padrão (DP) e erros padrão da estimativa (EPM) foram observados para a argila e a areia, ao passo que o silte apresentou o maior coeficiente de variação.

Tabela 1. Estatística descritiva do número de árvores $(\mathrm{N})$, área basal $(\mathrm{G})$, atributos químicos e granulométricos do solo, a 0 - 0,20 m de profundidade, em um remanescente de Floresta Ombrófila Mista.

Table 1. Descriptive statistics of number of trees $(\mathrm{N})$, basal area $(\mathrm{G})$, soil chemical attributes, and granulometry at $0-0,20 \mathrm{~m}$ of depth in a Mixed Ombrophilous Forest remnant.

\begin{tabular}{ccccccccccc}
\hline Variável & Grubbs & Min. & Med. & Max. & Md. & DP & EPM & CV & Assim. & KS \\
\hline $\mathrm{N}\left(\mathrm{n} \mathrm{ha}^{-1}\right)$ & $3,035^{\mathrm{ns}}$ & 192,00 & 566,64 & 1056,00 & 576,00 & 161,23 & 11,40 & 28,5 & 0,125 & $0,040^{\mathrm{ns}}$ \\
\hline
\end{tabular}




\begin{tabular}{ccccccccccc}
\hline${\mathrm{G}\left(\mathrm{m}^{2} \mathrm{ha}^{-1}\right)}^{2,645^{\mathrm{ns}}}$ & 7,40 & 30,40 & 53,60 & 29,60 & 8,80 & 0,60 & 28,9 & 0,310 & $0,079^{*}$ \\
\hline $\mathrm{pH}\left(\mathrm{em} \mathrm{CaCl}_{2}\right)$ & $3,746^{*}$ & 3,36 & 3,79 & 4,30 & 3,80 & 0,18 & 0,01 & 4,8 & 0,312 & $0,160^{*}$ \\
\hline $\mathrm{P}\left(\mathrm{mg} \mathrm{dm}^{-3}\right)$ & $6,419^{*}$ & 0,14 & 2,67 & 7,61 & 2,40 & 1,43 & 0,10 & 53,6 & 0,820 & $0,110^{*}$ \\
\hline $\mathrm{K}\left(\mathrm{cmol}_{\mathrm{c}} \mathrm{dm}^{-3}\right)$ & $5,758^{*}$ & 0,07 & 0,28 & 0,75 & 0,25 & 0,13 & 0,01 & 46,0 & 1,116 & $0,128^{*}$ \\
\hline $\mathrm{Ca}\left(\mathrm{cmol}_{\mathrm{c}} \mathrm{dm}^{-3}\right)$ & $4,529^{*}$ & 0,05 & 0,83 & 4,50 & 0,50 & 0,96 & 0,07 & 115,7 & 2,180 & $0,208^{*}$ \\
\hline $\mathrm{Mg}\left(\mathrm{cmol}_{\mathrm{c}} \mathrm{dm}^{-3}\right)$ & $4,318^{*}$ & 0,20 & 1,03 & 3,40 & 0,85 & 0,67 & 0,05 & 65,5 & 1,272 & $0,151^{*}$ \\
\hline $\mathrm{Al}\left(\mathrm{cmol}_{\mathrm{c}} \mathrm{dm}^{-3}\right)$ & $2,517^{\text {ns }}$ & 1,10 & 4,81 & 9,60 & 4,33 & 1,90 & 0,13 & 39,6 & 0,400 & $0,105^{*}$ \\
\hline $\mathrm{Al}+\mathrm{H}\left(\mathrm{cmol}_{\mathrm{c}} \mathrm{dm}^{-3}\right)$ & $3,189^{\text {ns }}$ & 8,29 & 16,77 & 29,25 & 16,33 & 3,91 & 0,28 & 23,3 & 0,612 & $0,094^{*}$ \\
\hline Argila $(\%)$ & $2,760^{\text {ns }}$ & 9,30 & 39,60 & 70,20 & 41,10 & 11,10 & 0,80 & 27,9 & $-0,232$ & $0,063^{\text {ns }}$ \\
\hline Areia $(\%)$ & $2,899^{\text {ns }}$ & 16,10 & 46,80 & 78,10 & 47,80 & 10,80 & 0,80 & 23,0 & $-0,090$ & $0,063^{\text {ns }}$ \\
\hline Silte $(\%)$ & $3,076^{\text {ns }}$ & 4,20 & 13,50 & 29,80 & 13,00 & 5,30 & 0,40 & 39,1 & 0,674 & $0,077^{*}$ \\
\hline
\end{tabular}

Min = mínimo; Med = média; Max = máximo; $\mathrm{Md}=$ mediana; $\mathrm{DP}=$ desvio padrão; $\mathrm{EPM}=$ erro padrão da média; $\mathrm{CV}=$ coeficiente de variação; Assim. = assimetria; $\mathrm{KS}=$ teste de Kolmogorov-Smirnov; ${ }^{\text {ns }}=$ não significativo (Teste de Grubbs = não há valores outliers na série de dados; e Teste KS = há distribuição normal); e * = significativo p<0,05 (Teste de Grubbs = há valores outliers; e Teste KS = não há distribuição normal).

O pH apresentou média de 3,8 e valores abaixo de 4,3, o que indicou acidez muito alta (RAIJ, 2011), ao passo que os teores médios de $\mathrm{P}$ foram considerados muitos baixos (BORTOLON; GIANELLO, 2008). Ao analisar a média de cada atributo, o nível de concentração de K foi considerado médio (CHAVES et al., 1998), enquanto os teores de $\mathrm{Ca}$ e $\mathrm{Mg}$ foram baixos (RAIJ, 2011). Contudo, o teor médio de $\mathrm{Al}$ foi superior a $1 \mathrm{cmol}_{\mathrm{c}} \mathrm{dm}^{-3}$ e classificado como alto e prejudicial à produção e desenvolvimento do sistema radicular das plantas (RAIJ, 2011).

Pelo teste de Kolmogorov-Smirnov, observou-se que somente o número de árvores $(\mathrm{N})$, a argila e a areia apresentaram distribuição normal, corroborando com os menores valores de assimetria, apesar de a normalidade não ser um pressuposto da geoestatística (YAMAMOTO; LANDIM, 2013). No entanto, as variáveis $\mathrm{P}, \mathrm{Al}+\mathrm{He}$ silte, que apresentaram coeficientes de assimetria entre 0,5 e 1,0 , foram transformadas em $\sqrt{x_{i}}$, ao passo que a transformação logarítmica foi aplicada para as variáveis que apresentaram assimetria maior que 1, entre elas $\mathrm{K}$, $\mathrm{Ca}$ e Mg. Essas transformações foram aplicadas seguindo recomendação de Webster e Oliver (2007).

Foi verificada a presença de dependência espacial para todas as variáveis, exceto para o atributo alumínio (Al), que não apresentou ajuste pelos modelos geoestatísticos (Tabela 2). Consequentemente, o modelo efeito pepita puro foi o escolhido. De maneira geral, foi observado grau forte de dependência espacial (GD) para o modelo exponencial, exceto para o número de árvores, $\mathrm{H}+\mathrm{Al}$, argila, areia e silte. $\mathrm{O}$ grau moderado de dependência espacial foi detectado para as demais variáveis.

Tabela 2. Parâmetros dos semivariogramas escalonados ajustados para o número de árvores (N), área basal $(\mathrm{G})$, atributos químicos e granulometria do solo, a 0 - 0,20 m de profundidade, em um remanescente de Floresta Ombrófila Mista.

Table 2. Parameters of scaled semivariograms fitted for the number of trees $(\mathrm{N})$, basal area $(\mathrm{G})$, soil chemical attributes, and granulometry at $0-0,20 \mathrm{~m}$ of depth in a Mixed Ombrophilous Forest remnant.

\begin{tabular}{cccccccc}
\hline Variável & Modelo & $\mathbf{C}_{\mathbf{0}}$ & $\mathbf{C}$ & $\mathbf{A}(\mathbf{m})$ & $\mathbf{G D}(\boldsymbol{\%})$ & $\mathbf{R}^{\mathbf{2}}$ & SQDP \\
\hline \multirow{3}{*}{$\mathrm{N}$} & Esférico & 0,343 & 0,633 & 164,1 & 35,2 & 0,802 & 0,0059 \\
\cline { 2 - 8 } & Exponencial & 0,050 & 0,929 & 141,0 & 5,1 & 0,744 & 0,0077 \\
\cline { 2 - 8 } & Gaussiano & 0,447 & 0,528 & 138,8 & 45,9 & 0,792 & 0,0063 \\
\hline \multirow{3}{*}{$\mathrm{G}$} & Esférico & 0,621 & 0,381 & 143,0 & 62,0 & 0,84 & 0,0017 \\
\cline { 2 - 8 } & Exponencial & 0,312 & 0,690 & 108,8 & 31,2 & 0,797 & 0,0020 \\
\cline { 2 - 8 } & Gaussiano & 0,680 & 0,316 & 128,3 & 68,3 & 0,823 & 0,0018 \\
\hline \multirow{3}{*}{$\mathrm{pH}$} & Esférico & 0,682 & 0,274 & 149,4 & 71,3 & 0,738 & 0,0012 \\
\cline { 2 - 8 } & Exponencial & 0,553 & 0,406 & 131,0 & 57,7 & 0,649 & 0,0016 \\
\hline \multirow{3}{*}{$\mathrm{P}$} & Gaussiano & 0,738 & 0,220 & 131,4 & 77,1 & 0,718 & 0,0013 \\
\hline & Esférico & 0,492 & 0,468 & 196,7 & 51,2 & 0,982 & 0,0004 \\
\hline & Exponencial & 0,316 & 0,670 & 198,3 & 32,0 & 0,981 & 0,0003 \\
\hline & Gaussiano & 0,595 & 0,377 & 186,7 & 61,2 & 0,984 & 0,0003 \\
\hline
\end{tabular}




\begin{tabular}{|c|c|c|c|c|c|c|c|}
\hline \multirow{3}{*}{ K } & Esférico & 0,672 & 0,242 & 219,3 & 73,5 & 0,562 & 0,0033 \\
\hline & Exponencial & 0,580 & 0,348 & 223,5 & 62,5 & 0,535 & 0,0033 \\
\hline & Gaussiano & 0,721 & 0,212 & 226,3 & 77,3 & 0,647 & 0,0027 \\
\hline \multirow{3}{*}{$\mathrm{Ca}$} & Esférico & 0,611 & 0,358 & 230,2 & 63,0 & 0,699 & 0,0046 \\
\hline & Exponencial & 0,461 & 0,523 & 223,2 & 46,9 & 0,679 & 0,0046 \\
\hline & Gaussiano & 0,684 & 0,302 & 226,3 & 69,3 & 0,753 & 0,0038 \\
\hline \multirow{3}{*}{$\mathrm{Mg}$} & Esférico & 0,780 & 0,195 & 210,2 & 80,0 & 0,682 & 0,0012 \\
\hline & Exponencial & 0,697 & 0,286 & 203,2 & 70,9 & 0,647 & 0,0012 \\
\hline & Gaussiano & 0,820 & 0,165 & 208,4 & 83,2 & 0,748 & 0,0010 \\
\hline \multirow{3}{*}{$\mathrm{Al}$} & Esférico & 0,736 & - & - & - & - & - \\
\hline & Exponencial & 0,756 & - & - & - & - & - \\
\hline & Gaussiano & 0,748 & - & - & - & - & - \\
\hline \multirow{3}{*}{$\mathrm{H}+\mathrm{Al}$} & Esférico & 0,417 & 0,576 & 212,6 & 42,0 & 0,946 & 0,0014 \\
\hline & Exponencial & 0,168 & 0,850 & 205,3 & 16,5 & 0,934 & 0,0013 \\
\hline & Gaussiano & 0,542 & 0,470 & 203,8 & 53,5 & 0,958 & 0,0011 \\
\hline \multirow{3}{*}{ Argila } & Esférico & 0,219 & 0,862 & 228,6 & 20,3 & 0,985 & 0,0009 \\
\hline & Exponencial & 0,050 & 1,040 & 225,8 & 4,6 & 0,951 & 0,0029 \\
\hline & Gaussiano & 0,360 & 0,770 & 223,6 & 31,8 & 0,980 & 0,0011 \\
\hline \multirow{3}{*}{ Areia } & Esférico & 0,321 & 0,777 & 199,2 & 29,2 & 0,983 & 0,0006 \\
\hline & Exponencial & 0,147 & 1,015 & 240,5 & 12,7 & 0,953 & 0,0020 \\
\hline & Gaussiano & 0,471 & 0,637 & 179,4 & 42,5 & 0,985 & 0,0007 \\
\hline \multirow{3}{*}{ Silte } & Esférico & 0,428 & 0,667 & 258,0 & 39,1 & 0,990 & 0,0004 \\
\hline & Exponencial & 0,181 & 0,944 & 256,3 & 16,1 & 0,982 & 0,0006 \\
\hline & Gaussiano & 0,564 & 0,552 & 243,4 & 50,6 & 0,982 & 0,0005 \\
\hline
\end{tabular}

Em que: $\mathrm{C}_{\mathrm{o}}=$ efeito pepita; $\mathrm{C}=$ contribuição (variância estrutural); $\mathrm{A}=$ alcance; $\mathrm{GD}=$ grau de dependência espacial; $\mathrm{R}^{2}=$ coeficiente de determinação; e SQDP = soma de quadrados dos desvios ponderados escalonados.

Os valores dos coeficientes de determinação $\left(\mathrm{R}^{2}\right)$ foram superiores a 0,9 para os atributos $\mathrm{P}, \mathrm{H}+\mathrm{Al}$, argila, areia e silte (Tabela 2), os quais também apresentaram os menores valores de soma de quadrados dos desvios ponderados (SQDP). Em contrapartida, os menores $\mathrm{R}^{2}$ e os maiores SQDP foram observados para as demais variáveis. Os modelos esférico e gaussiano proporcionaram os melhores ajustes aos semivariogramas experimentais, com os quais foram obtidos os menores (SQDP) e os maiores $\mathrm{R}^{2}$, conforme a validação cruzada (Tabela 3).

Tabela 3. Parâmetros da validação cruzada dos ajustes geoestatísticos selecionados para o número de árvores (N), área basal $(\mathrm{G})$, atributos químicos e granulometria do solo, a $0-0,20 \mathrm{~m}$ de profundidade, em um remanescente de Floresta Ombrófila Mista.

Table 3. Cross validation parameters of geostatistical fits selected for the number of trees $(\mathrm{N})$, basal area $(\mathrm{G})$, soil chemical attributes, and granulometry at $0-0,20 \mathrm{~m}$ of depth in a Mixed Ombrophilous Forest remnant.

\begin{tabular}{cccccc}
\hline \multirow{2}{*}{ Variável } & \multirow{2}{*}{ Modelo Selecionado } & \multicolumn{2}{c}{ Coeficiente } & \multirow{2}{*}{$\mathbf{R}_{\mathbf{v c}}$} & \multirow{2}{*}{$\mathbf{S}_{\mathbf{y x}} \boldsymbol{}$} \\
\cline { 3 - 4 } & & Linear & Angular & & 0,399 \\
\hline $\mathrm{N}$ & Esférico & 3,033 & 0,994 & 22 \\
\hline $\mathrm{G}$ & Esférico & 5,074 & 0,833 & 0,119 & 27,1 \\
\hline $\mathrm{pH}$ & Esférico & 0,234 & 0,937 & 0,184 & 4,4 \\
\hline $\mathrm{P}$ & Gaussiano & 0,460 & 0,87 & 0,229 & 46,9 \\
\hline $\mathrm{K}$ & Gaussiano & 0,076 & 0,71 & 0,091 & 43,7 \\
\hline $\mathrm{Ca}$ & Gaussiano & 0,36 & 0,536 & 0,019 & 114,2 \\
\hline $\mathrm{Mg}$ & Gaussiano & 0,400 & 0,588 & 0,023 & 64,5 \\
\hline $\mathrm{Al}$ & Efeito pepita puro & 0,673 & - & - & - \\
\hline $\mathrm{H}+\mathrm{Al}$ & Gaussiano & 0,380 & 0,986 & 0,35 & 18,8 \\
\hline $\mathrm{Argila}$ & Esférico & 0,976 & 0,925 & 0,514 & 19,4 \\
\hline
\end{tabular}




\begin{tabular}{cccccc}
\hline Areia & Esférico & 2,414 & 0,95 & 0,38 & 18,1 \\
\hline Silte & Esférico & $-0,350$ & 1,049 & 0,415 & 29,9 \\
\hline
\end{tabular}

Em que: $\mathrm{R}_{\mathrm{vc}}^{2}=$ coeficiente de determinação da validação cruzada; e $\mathrm{S}_{\mathrm{yx}} \%$ = erro padrão da estimativa em porcentagem.

Os ajustes selecionados para as características edáficas e da floresta resultaram em coeficientes lineares de $-0,350$ a 5,074; coeficientes angulares entre 0,536 a 1,049; coeficientes de determinação da validação cruzada $\left(\mathrm{R}_{\mathrm{vc}}{ }_{\mathrm{v}}\right)$ de 0,019 e 0,514 ; e erros padrão estimativa $\left(\mathrm{S}_{\mathrm{yx}} \%\right)$ de 4,4 a $114,2 \%$ (Tabela 3$)$. Apesar do elevado $\mathrm{S}_{\mathrm{yx}} \%$, a validação cruzada não visa rejeitar um modelo de semivariograma, sendo uma técnica adequada para identificar erros ou problemas na base de dados (ANDRIOTTI, 2003). Os atributos químicos P, K, Ca e Mg apresentaram os menores coeficientes angulares e $\mathrm{R}^{2}{ }_{\mathrm{vc}}$ baixos, além dos maiores $\mathrm{S}_{\mathrm{yx}} \%$. Para as demais variáveis, os coeficientes lineares e angulares foram muito próximos aos ideais teóricos, com menor $\mathrm{S}_{\mathrm{yx}} \%$ para o $\mathrm{pH}$, quando comparado às demais variáveis.

Com os semivariogramas designados pela validação cruzada, observou-se dispersão reduzida dos valores observados junto da linha média estimada para a granulometria do solo, bem como para $\mathrm{P}, \mathrm{Mg}$ e $\mathrm{H}+\mathrm{Al}$, sendo o maior espalhamento observado para o número de árvores, área basal, $\mathrm{pH}, \mathrm{K}$ e $\mathrm{Ca}$ (Figura 2). Para o atributo $\mathrm{Al}$, não foi observada correlação espacial entre os pontos amostrados, revelando um comportamento semelhante ao modelo efeito pepita puro, os quais são semivariogramas que carecem de estrutura espacial, em que o valor de semivariância é constante em todas as distâncias (YAMAMOTO; LANDIM, 2013). Entre os atributos do solo e da floresta, o efeito pepita $\left(\mathrm{C}_{0}\right)$ apresentou valores inferiores a 0,5 (Figura 2) para o número de árvores, argila e areia, à medida que $\mathrm{pH}, \mathrm{K}, \mathrm{Ca}$ e $\mathrm{Mg}$ apresentaram os maiores valores de $\mathrm{C}_{0}$. Isso indicou possibilidade de maior erro nas estimativas desses atributos (ISAAKS; SRIVASTAVA, 1989). Ademais, o comportamento isotrópico foi admitido em decorrência da semelhança dos semivariogramas direcionais no plano espacial.
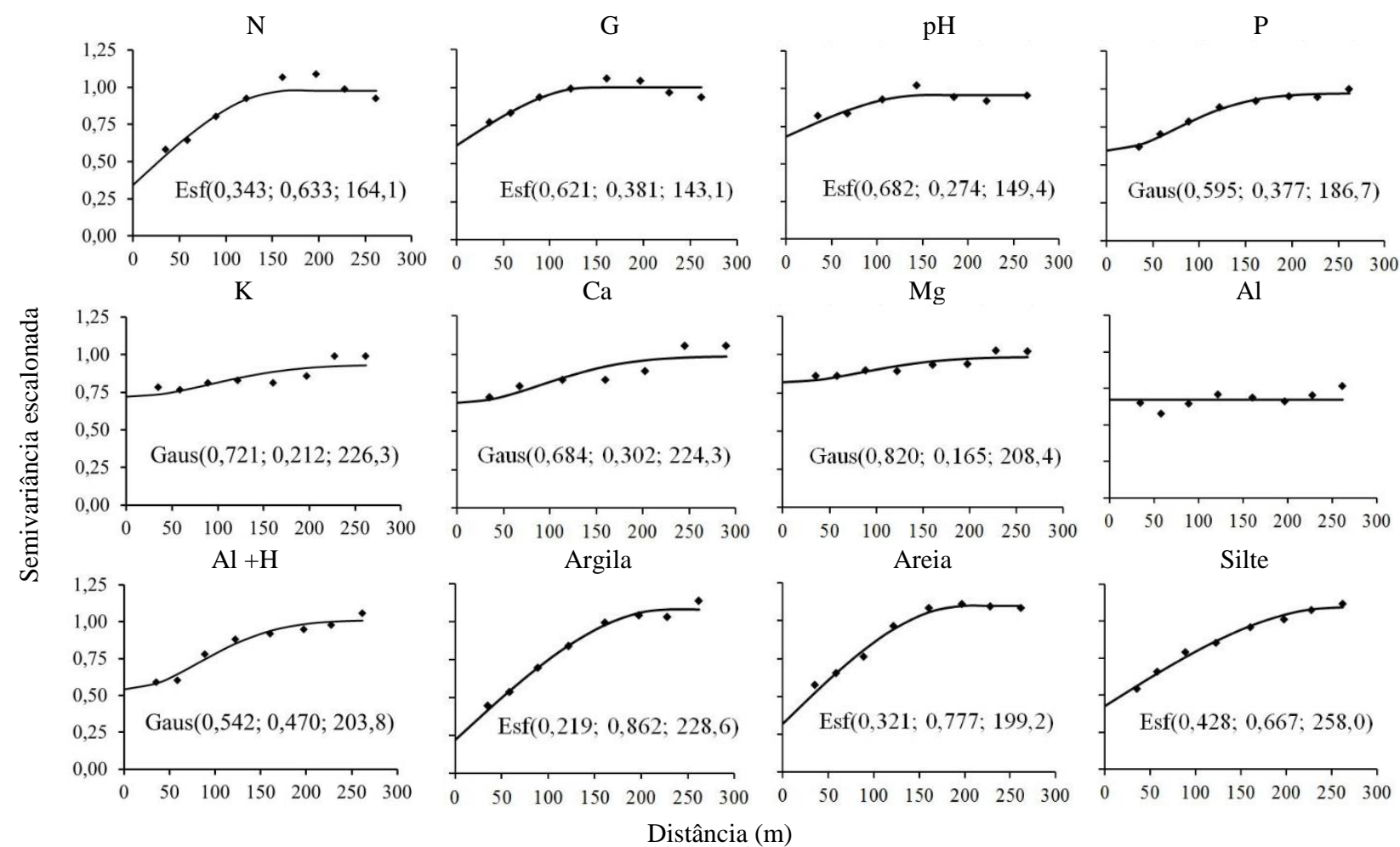

Figura 2. Semivariogramas teóricos ajustados para o número de árvores (N), área basal (G), atributos químicos e granulometria do solo, a 0 - 0,20 m de profundidade, em um remanescente de Floresta Ombrófila Mista.

Figure 2. Theoretical semivariograms fitted for the number of trees $(\mathrm{N})$, basal area $(\mathrm{G})$, soil chemical attributes, and granulometry at 0 - 20m of depth in a Mixed Ombrophilous Forest remnant.

Com o auxílio dos mapas temáticos do número de árvores (Figura 3A), área basal (Figura 3B), pH (Figura 3C), P (Figura 3D), K (Figura 3E), Ca (Figura 3F), Mg (Figura 3G), H + Al (Figura 3H), argila (Figura 3I), silte (Figura 3J) e areia (Figura 3K) obtidos após a krigagem ordinária pontual, foram observados padrões distintos e grande variabilidade para todas as características edáficas e da floresta, apesar de o número de árvores e área basal 
não apresentarem expressiva relação espacial com os atributos dos solos. Contudo, uma sutil similaridade espacial foi observada nos locais com menor $\mathrm{pH}$ (solos mais ácidos) e com valores inferiores do número de árvores e da área basal, bem como nas áreas com menores concentrações de $\mathrm{H}+\mathrm{Al}$, as quais apontaram para uma leve tendência de maior estoque da floresta.

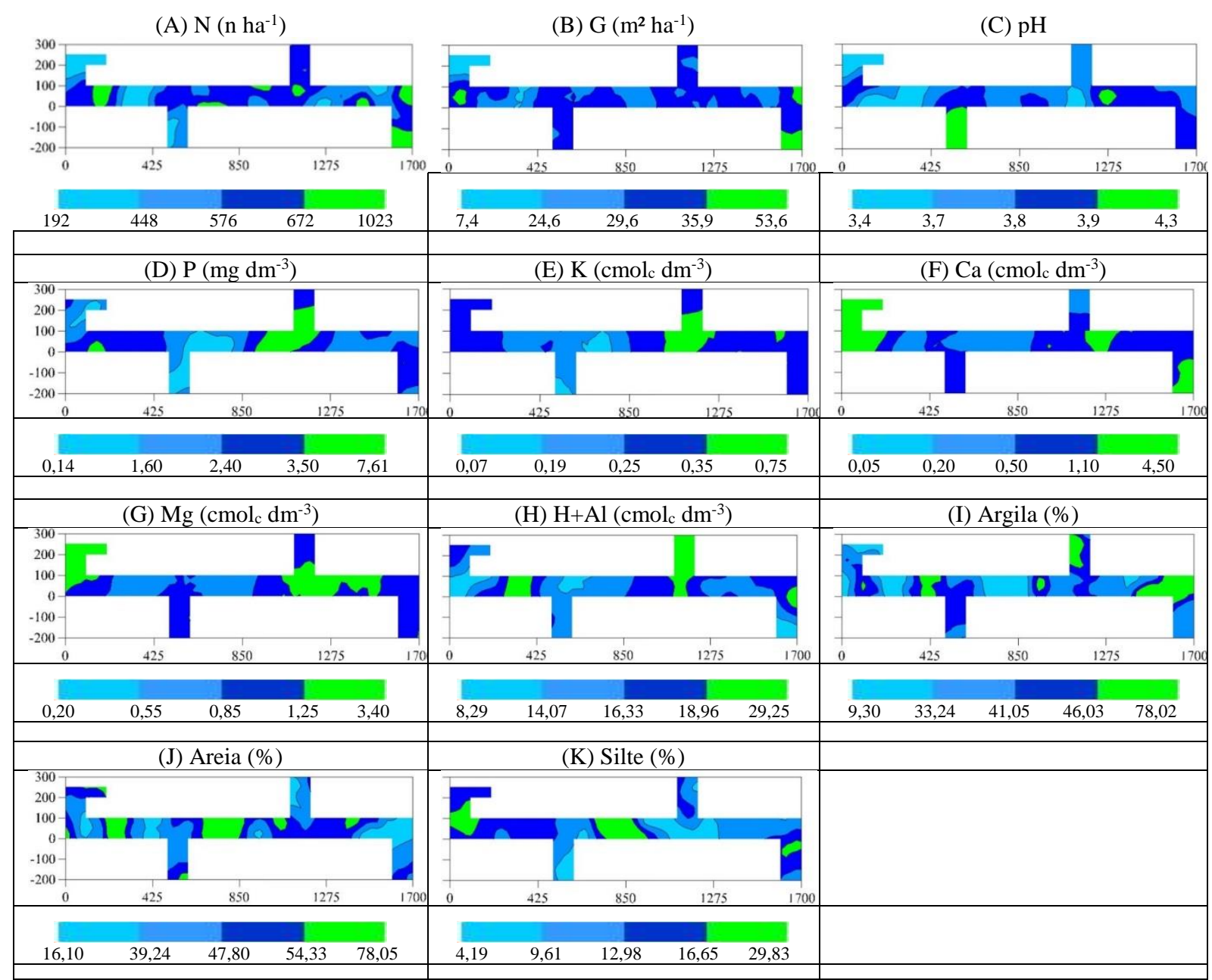

Figura 3. Mapas temáticos obtidos pela krigagem ordinária pontual para o número de árvores (N), área basal $(\mathrm{G})$, atributos químicos e granulometria do solo, a 0 - 0,20 $\mathrm{m}$ de profundidade, em um remanescente de Floresta Ombrófila Mista.

Figure 3. Thematic maps obtained by punctual ordinary kriging for the number of trees $(\mathrm{N})$, basal area $(\mathrm{G})$, soil chemical attributes, and granulometry at $0-0,20 \mathrm{~m}$ of depth in a Mixed Ombrophilous Forest remnant.

Quando as características edáficas e da floresta foram analisadas separadamente, observou-se relação espacial entre os mapas temáticos. O número de árvores e a área basal apresentaram elevada semelhança entre os mapas temáticos (Figura 3A e 3B), sendo as variáveis diretamente proporcionais, com predomínio da classe III, cujos intervalos oscilaram entre 576 a $672 \mathrm{n} \mathrm{ha}^{-1}$ e 29,6 a 35,9 $\mathrm{m}^{2} \mathrm{ha}^{-1}$, para o número de árvores e a área basal, respectivamente.

Para a textura do solo, a maior relação foi observada entre as variáveis argila (Figura 3J) e areia (Figura $3 \mathrm{~K}$ ), sendo inversamente proporcionais entre os mapas temáticos, cujos locais que apresentaram menor quantidade de argila, entre 8,29 a 14,07\%, exibiram a maior concentração de areia, entre 54,33 a 78,05\%. Cabe salientar que a soma das frações areia, silte e argila, quando realizadas individualmente nos mapas, não garantem a estimativa de $100 \%$. Ademais, entre as variáveis químicas, pH (Figura 3C) e H $+\mathrm{Al}$ (Figura 3H) mostraram relação inversa, em que os locais com menor valor de $\mathrm{pH}$, entre 3,4 a 3,7, apresentaram a tendência de valores médios a elevados de $\mathrm{H}+\mathrm{Al}$, entre 19,33 e $29,25 \mathrm{cmol}_{\mathrm{c}} \mathrm{dm}^{-3}$. 


\section{DISCUSSÃO}

A média aritmética dos atributos químicos (Tabela 1), a elevada acidez, a alta concentração de $\mathrm{Al}$ e a baixa soma de bases $(\mathrm{K}+\mathrm{Ca}+\mathrm{Mg})$ indicaram solos com baixa disponibilidade de nutrientes distribuídos em algumas glebas na área de estudo, uma vez que a maioria dos nutrientes não se encontra na forma solúvel e passível de serem absorvidos com o pH entre 5,6 e 6,2 (COSTA; ZOCCHE, 2009). Esses resultados foram semelhantes aos observados em outros estudos em áreas de floresta com araucária (RODRIGUES et al., 2016).

A acidez do solo das florestas nativas está relacionada com a mineralização da matéria orgânica e aos exsudatos ácidos liberados pelas raízes das plantas (BARRETO et al., 2006). Segundo Rodrigues et al. (2016), em condições de elevada acidez, os compostos de alumínio, ferro e manganês tornam-se bastante solúveis e, além de causarem toxidez às plantas, são responsáveis pela fixação de fósforo e pela formação de compostos de fósforo insolúveis na solução do solo, reduzindo significativamente a disponibilidade desse nutriente às plantas. Em função disso, a baixa taxa de incremento em florestas nativas pode estar relacionada à acidificação do solo.

Em relação à textura do solo, os resultados demonstraram o predomínio das frações areia e argila para a área de estudo (Tabela 1). Segundo a Embrapa (2013), com base nas proporções entre areia, silte e argila, a textura do solo é definida como muito argilosa (solos com mais de 60\% de argila), argilosa (solos com 35 a $60 \%$ de argila) e média (solos com menos de 35\% de argila e mais de 15\% de areia). A variação da composição de solos arenosos e argilosos influencia na disponibilidade de água para as plantas à medida que a retenção de água é maior em solos argilosos e, portanto, há maior disponibilidade para as plantas (REINERT; REICHERT, 2006).

Com relação à continuidade espacial do número de árvores, área basal e atributos do solo, os resultados corroboraram que o remanescente de Floresta Ombrófila Mista apresenta dependência espacial possível de ser modelada (Tabela 2), com dependência espacial (GD) moderada a forte para os ajustes geoestatísticos, exceto para o Al que não apresentou continuidade espacial. Segundo Andriotti (2003), o comportamento encontrado para o Al indica a ausência completa de correlação espacial entre os pontos amostrados e, nesse caso, os resultados geoestatísticos são os mesmos indicados pela estatística clássica.

Com a modelagem geoestatística, foram observados valores diferenciados nos parâmetros da validação cruzada (Tabela 3), com superioridade para o ajuste com o modelo esférico e gaussiano. O modelo esférico é predominante em trabalhos de ciência do solo, corroborando com a descrição de superioridade nos ajustes de atributos do solo e de plantas (SIQUEIRA et al., 2008), enquanto o modelo gaussiano tem comportamento parabólico na vizinhança e reflete uma grande continuidade da variável ajustada (ANDRIOTTI, 2003).

Com auxílio dos mapas temáticos, observou-se que o remanescente florestal apresenta considerável heterogeneidade espacial para o número de árvores, área basal e atributos do solo (Figura 3), corroborando com a variabilidade numérica das variáveis ao longo do espaço. Com isso, o ato de assumir apenas um valor de medida de posição central não é adequado para representar toda a extensão da área, conforme relatado por Rufino et al. (2006).

A comparação visual entre os mapas de variabilidade espacial da textura do solo e das variáveis dendrométricas revelou a ausência de um padrão de comportamento entre as variáveis (Figura 3). No entanto, evidenciou-se que os menores números de árvores e estoques de área basal se concentraram nas áreas com solos ácidos, ao passo que os locais com menores concentrações de $\mathrm{H}+\mathrm{Al}$ apontaram leve tendência de maior estoque da floresta. A influência do pH sobre os atributos físico-químicos do solo e da floresta, como área basal, número de indivíduos e índice de diversidade de Shannon, foi relatado por Rodrigues et al. (2016) em uma Floresta Ombrófila Mista Aluvial no município de Guarapuava, estado do Paraná.

Apesar da baixa relação espacial do número de árvores e área basal com a granulometria do solo, alguns autores relacionaram a ocorrência e a densidade de determinadas espécies ao nível de areia e argila, destacandose o trabalho de Almeida-Scabbia et al. (2011) em Floresta Estacional Semidecidual. Além disso, Rodrigues et al. (2016) também observaram forte correlação entre as variáveis ambientais e a ocorrência de determinadas espécies em um remanescente de Floresta Ombrófila Mista Aluvial.

Portanto, a baixa correlação observada entre os atributos do solo com o número de árvores e a área basal para o remanescente de floresta nativa revela que a heterogeneidade das variáveis dendrométricas não é explicada por uma única variável da textura do solo ou atributo químico. Nesse contexto, existem dificuldades na aplicação de metodologias que investiguem a interação de todos os fatores bióticos e abióticos que influenciam na produção das florestas nativas. Entretanto, o uso da geoestatística é válido para modelar, mapear e identificar tendências do potencial produtivo das florestas e dos fatores do ambiente.

\section{CONCLUSÕES}

- Os atributos do solo e da floresta nativa apresentam dependência espacial, seguindo padrões espaciais bem definidos e possibilitando detalhar a variabilidade espacial por meio da modelagem geoestatística. 
- Há ausência de forte similaridade espacial entre os mapas de krigagem dos atributos do solo com as variáveis número de árvores e área basal da floresta, porém evidencia-se que o pH influencia na disponibilidade de nutrientes e na estrutura arbórea da floresta nativa.

\section{REFERÊNCIAS}

ALMEIDA-SCABBIA, R. J.; SCHLLITLER, F. H. M.; CESAR, O.; MONTEIRO, R.; GOMES, E. P. C.; ROMANIUC NETO, S. Características físico-químicas do solo e distribuição de espécies arbóreas. Revista Brasileira de Biociências, v. 9, n. 3, p. 322-331, 2011.

ANDRIOTTI, J. L. S. Fundamentos de estatística e geoestatística. São Leopoldo: UNISINOS, 2003, 165 p.

BARRETO, A. C.; LIMA, F. H. S.; FREIRE, M. B. G. S.; ARAUJO, Q. R.; FREIRE, F. J. Características químicas e físicas de um solo sob floresta, sistema agroflorestal e pastagem no sul da Bahia. Caatinga, v.19, n. 4, p. 415425, 2006.

BORTOLON, L.; GIANELLO, C. Interpretação de resultados analíticos de fósforo pelos extratores Mehlich-1 e Mehlich-3 em solos do Rio Grande do Sul. Revista Brasileira de Ciência de Solo, v. 32, p. 2751-2756, 2008.

CAMBARDELLA, C. A.; MOORMAN, T. B.; NOVAK, J. M.; PARKIN, T. B.; KARLEN, D. L.; TURCO, R. F.; KONOPKA, A. E. Field-scale variability of soil properties in central Iowa soils. Soil Science Society of America Journal, v. 58, n. 5, p. 1501-1511, 1994.

CARVALHO, W. A. C.; OLIVEIRA FILHO, A. T.; FONTES, M. A. L.; CURI, N. Variação espacial da estrutura da comunidade arbórea de um fragmento de floresta semidecídua em Piedade do Rio Grande, MG, Brasil. Revista Brasileira de Botânica, v. 30, n. 2, p. 315-335, 2007.

CHAVES, L. H. G.; MENINO, I. B.; ARAÚJO, I. A.; CHAVES, I. B. Avaliação da fertilidade dos solos das várzeas do município de Sousa, PB. Revista Brasileira de Engenharia Agrícola e Ambiental, v. 2, n. 3, p. 262267, 1998.

COSTA, S.; ZOCCHE, J. J. Fertilidade de solos construídos em áreas de mineração de carvão na Região Sul de Santa Catarina. Revista Árvore, v. 33, n. 4, p. 665-674, 2009.

EMBRAPA - Empresa Brasileira de Pesquisa Agropecuária. Sistema brasileiro de classificação de solos. Brasília: Embrapa, 3 ed. 2013, 353 p.

GERHARDT, E. J.; FINGER, C. A. G.; LONGHI, S. J.; SCHUMACHER, M. V. Contribuição da análise multivariada na classificação de sítios em povoamentos de Araucaria angustifolia (Bert.) O. Ktze., baseada nos fatores físicos e morfológicos do solo e no conteúdo de nutrientes da serapilheira. Ciência Florestal, v. 11, n. 2, p. 41-57, 2001.

ISAAKS, E. H.; SRIVASTAVA, R. M. An introduction to applied geoestatistics. Oxford University Press, $1989,561 \mathrm{p}$.

KITAMURA, A. E.; ROCHA LIMA, C. G.; PASSOS, E.; CARVALHO, M. Relação entre a variabilidade espacial das frações granulométricas do solo e a produtividade do feijoeiro sob plantio direto. Revista Brasileira de Ciência do Solo, v. 31, n. 2, p. 361-369, 2007.

LOPES, L. C. M.; MARIANO-NETO, E.; AMORIN, A. M. Can soil types explain species distributions? Evaluating the woody understory component of a tropical forest in Brazil. Brazilian Journal of Botany, v. 39, n. 1, p. 251-259, 2016.

RAIJ, B. van. Fertilidade do solo e manejo de nutrientes. Piracicaba: International Plant Nutrition Institute. 2011, 420 p.

REINERT, D. J.; REICHERT, J. M. Propriedades físicas do solo. Universidade Federal de Santa Maria, 2006, $18 \mathrm{p}$.

RODRIGUES, A. L.; WATZLAWICK, L. F.; GENÚ, A. M.; HESS, A. F.; EBLING, A. A. Atributos de um solo florestal em uma topossequência e relações com a comunidade arbórea. Floresta, v. 46, n. 2, p. 145-154, 2016.

ROVEDDER, A. P. M.; ALMEIDA, C. M.; ARAUJO, M. M.; TONETTO, T. S.; SCOTTI, M. S. V. Relação solovegetação em remanescente da floresta estacional decidual na Região Central do Rio Grande do Sul. Ciência Rural, v. 44, n. 12, p. 2178-2185, 2014. 
RUFINO, T. M. C.; THIERSCH, C. R.; FERREIRA, S. O.; KANEGAE JUNIOR, H.; FAIS, D. Uso da Geoestatística no estudo da relação entre variáveis dentrométricas de povoamentos de Eucalyptus sp. e atributos do solo. Ambiência, v. 2, n. 1, p. 83-93, 2006.

SANTOS, M. C. N.; MELlO, J. M.; MELLO, C. R.; AVILA, L. F. Spatial continuity of soil attributes in an atlantic forest remnant in the mantiqueira range, MG. Ciência e Agrotecnologia, v. 37, n. 1, p. 68-77, 2013

SANTOS, R. D; LEMOS, R. C.; SANTOS, H. G.; KER, J. C.; ANJOS, L. H. C.; SHIMIZU, S. H. Manual de Descrição e Coleta de Solos no Campo. Viçosa: SBCS, 6 ed. 2013b, 100 p.

SIQUEIRA, G. M.; VIEIRA, S. R.; CEDDIA, M. B. Variabilidade espacial de atributos físicos do solo determinados por métodos diversos. Bragantia, v. 67, p. 203-211, 2008.

SILVA, A. M.; CANUTO, D. S. O.; MORAES, M. L. T.; BUZETTI, S. Avaliação das propriedades químicas em solo de cerrado sob reflorestamento ciliar. Floresta, v. 42, n. 1, p. 49-58, 2012.

TOLEDO, L; ANJOS, L. H. C.; COUTO, W. H.; CORREIA, J. R.; PEREIRA, M. G.; CORREIA, M. E. F. Análise multivariada de atributos pedológicos e fitossociológicos aplicada na caracterização de ambientes de cerrado no norte de minas gerais. Revista Árvore, v. 33, n. 5, p. 957-968, 2009.

YAMAMOTO, J. K.; LANDIM, P. M. B. Geoestatística: conceitos e aplicações. São Paulo: Oficina de textos, 2013, $215 \mathrm{p}$.

WEBSTER, R.; OLIVER M. A. Geostatistics for Environmental Scientists. Chichester: John Wiley \& Sons, 2 ed. $2007,325 \mathrm{p}$.

VIEIRA, S. R.; NIELSEN, D. R.; BIGGAR, J. W.; TILLOTSON, P. M. The Scaling of semivariograms and the kriging estimation of field-measured properties. Revista Brasileira de Ciência do Solo, v. 21, p. 525-533, 1997. 